\title{
Diagnóstico de autismo no século XXI: evolução dos domínios nas categorizações nosológicas
}

\author{
Conceição Santos Fernandes ${ }^{\text {* }}$ (i) \\ Jeane Tomazellib ${ }^{\circ}$ \\ Vania Reis Girianellic $\mathbb{C}$ \\ aPontifícia Universidade Católica do Rio de Janeiro. Rio de Janeiro, RJ, Brasil \\ ${ }^{b}$ Instituto Nacional de Câncer José Alencar Gomes da Silva. Rio de Janeiro, RJ, Brasil \\ 'Escola Nacional de Saúde Pública. Rio de Janeiro, RJ, Brasil
}

\begin{abstract}
Resumo: Os critérios que subsidiaram o diagnóstico do autismo passaram por diversas mudanças ao longo dos anos, acompanhando os pressupostos conceituais hegemônicos no período. 0 objetivo deste estudo é analisar a evolução do diagnóstico do autismo no século XXI, a partir dos domínios e subdomínios em que se baseiam as categorizações nosológicas. Adicionalmente, são sinalizados instrumentos auxiliares utilizados e algumas tecnologias diagnósticas em desenvolvimento, além de modelos conceituais que tratam do perfil neuropsicológico. Trata-se de pesquisa documental, tendo como referência os manuais diagnósticos existentes no período. Os domínios de interação social, comunicação e padrão restrito e repetitivo de comportamento foram mantidos nos diferentes manuais diagnósticos abordados. Os subdomínios de interação social e comunicação foram reduzidos, enquanto o padrão restrito e repetitivo teve consolidação no DSM-5. Compreender a evolução dos critérios diagnósticos tende a promover o desenvolvimento da clínica, potencializando a antecipação do diagnóstico e as intervenções necessárias para um melhor prognóstico.
\end{abstract}

Palavras-chave: Transtorno do Espectro Autista, Diagnóstico, Manual Diagnóstico e Estatístico de Transtornos Mentais, Classificação Internacional de Doenças.

\section{Introdução}

O autismo clássico foi descrito pela primeira vez por Leo Kanner, em 1943, como "Distúrbios autísticos do contato afetivo", a partir da análise de onze casos com patologia grave e condições singulares; que englobava, além da inabilidade para estabelecer contato afetivo, comportamentos obsessivos, ecolalia e estereotipia (Kanner, 1943). Ele, no entanto, apresentou indicações ambíguas quanto à origem do autismo: articulação com a personalidade dos pais e o tipo de relações precoces estabelecidas entre eles e as crianças; forma precoce de esquizofrenia; e entidade nosológica específica diferente da esquizofrenia infantil, pois o rompimento com a realidade ocorria desde o início da vida. A hipótese de incapacidade inata abriu espaço para concepção organicista, cuja origem das doenças está relacionada à disfunção de natureza bioquímica, genética ou neuropsicológica (Facion, 2005).

Os critérios que subsidiaram o diagnóstico do autismo passaram por diversas mudanças ao longo dos anos e foram descritos nos manuais de categorização nosológica. Os mais conhecidos e utilizados são o Manual Diagnóstico e Estatístico de Transtornos Mentais (DSM) e a Classificação Internacional de Doenças e Problemas

* Endereço para correspondência: conceicaosf@yahoo.com.br
Relacionados à Saúde (CID), particularmente a partir da década de 1980. Estes manuais apresentam diferenças nas nomenclaturas, características e códigos utilizados para fins de diagnóstico, mas convergem nos pressupostos conceituais que embasaram a classificação nosológica, hegemônicos no período em que foram publicados. A principal mudança, contudo, foi a substituição da perspectiva psicanalítica da doença mental, cuja origem seria provocada por eventos traumáticos e fundamentada nos conceitos de personalidade, estrutura e psicodinâmica, pelo modelo biomédico com diagnóstico categórico (agrupamento de sintomas) e abordagem multiaxial, que considera também a dimensão orgânica e a influência dos fatores externos sobre o comportamento (Dunker, 2014). Esse processo foi acompanhado pelo surgimento e crescimento da indústria farmacêutica, em particular dos psicofármacos, com consequente aumento do diagnóstico das doenças mentais (Aguiar \& Ortega, 2017; Whitaker, 2017).

A primeira edição do DSM, manual da Associação Americana de Psiquiatria (APA), foi desenvolvida a partir dos sistemas de coleta de recenseamento e estatísticas de hospitais psiquiátricos e do manual do Exército dos Estados Unidos, utilizado para seleção e acompanhamento de recrutas. Também sofreu influência do sistema diagnóstico de Adolf Meyer com enfoque na oposição entre neurose e psicose (Dunker, 2014), e o autismo foi classificado como 
sintoma da "Reação Esquizofrênica tipo Infantill" (American Psychiatric Association, 1952). Na segunda edição, o autismo foi denominado "Esquizofrenia tipo Infantil" (American Psychiatric Association, 1968), mas sem apresentar alterações significativas em relação à edição anterior.

A primeira sistematização do autismo na CID ocorreu na sexta classificação, implantada em 1950, quando passou a ser coordenada pela Organização Mundial da Saúde (OMS). $\mathrm{Na}$ CID-6, o número de categorias foi ampliado passando a incluir doenças não fatais e teve uma seção exclusiva para classificar os transtornos mentais (Benedicto, Wai, Oliveira, Godoy, \& Costa, 2013). Na época, o autismo foi inserido na categoria "Perturbações esquizofrênicas", mantendo-se associado à esquizofrenia até a nona edição (1979), com a nomenclatura "Psicose Infantil" ou "Síndrome de Kanner" (Vargas \& Schmidt, 2011).

Em 1977, a APA convocou um grupo de trabalho liderado pelo psiquiatra Robert Spitzer, tendo como diretriz estabelecer critérios descritivos e sintomas observáveis, fundamentados em critérios da medicina baseada em evidências (Dunker, 2014). O termo doença foi substituído por desordem, e o DSM-III foi lançado em 1980, constituindo-se uma mudança de paradigma ao iniciar o rompimento com a psicanálise (Martinhago \& Caponi, 2019). O autismo passa a ter designação própria: "Transtorno Autista", incluído nos transtornos invasivos de desenvolvimento (TID). Os critérios empregados refletiam as manifestações inicialmente descritas por Kanner e expandidas por Rutter (Stelzer, 2010). A décima versão da CID, publicada em 1989, adotou a terminologia autismo infantil e autismo atípico nos Transtornos Globais do Desenvolvimento (TGD), sob o código F84.

A partir da década de 1990, o DSM-IV ganhou uma perspectiva mais clara de desenvolvimento, mostrando relações com déficit cognitivo (Assumpção \& Pimentel, 2000; Gadia, Tuchman, \& Rotta, 2004), e se aproximou da padronização da CID-10. O Manual foi revisado anos depois (DSM IV-TR), trazendo pequenas alterações.

Na primeira década do século XXI, foram realizadas diversas conferências para levantamento da literatura de transtornos mentais produzida até então. Diferentes grupos de trabalho contribuíram para o desenvolvimento da quinta edição do DSM (American Psychiatric Association, 2014). O DSM-5 rompe com modelo multiaxial, embora mantenha a recomendação de avaliar fatores psicossociais e ambientais; também é retirada a escala de Avaliação Global do Funcionamento, mas sem contraindicar a utilização de diversas escalas para auxiliar o diagnóstico. Nessa categorização nosológica, o autismo passa a ser considerado um transtorno do neurodesenvolvimento (Araújo \& Neto, 2014) e denominado transtornos do espectro autista (TEA). Essa categoria absorve em um único diagnóstico os outros transtornos especificados no transtornos invasivos de desenvolvimento (TID), fazendo apenas distinção quanto ao nível de gravidade em relação à interação e comunicação. O diagnóstico é clínico, feito por indicadores, por meio de observações comportamentais e relatos quanto ao histórico do desenvolvimento, guiado por critérios universais e descritivos com base em teorias do desenvolvimento e das neurociências (Marques \& Bosa, 2015; Neumann et al., 2017). Cabe a ressalva de que, para transtorno de Rett já existe a identificação de mutação genética ainda que outros fatores possam estar atuando na expressão da doença (Huppke, Laccome, Krämer, Engel, \& Hanefeld, 2000). O CID-11, publicado em 2018, mantém a terminologia (TEA) e as alterações realizadas no DSM-5, mas com redução dos subdomínios que embasam o diagnóstico.

Objetivo deste estudo é analisar a evolução do diagnóstico do autismo no século XXI, a partir dos domínios e subdomínios em que se baseiam as categorizações nosológicas existentes no período.

\section{Percurso metodológico}

Trata-se de pesquisa documental sobre a evolução do diagnóstico do autismo no século XXI, tendo como referência os manuais diagnósticos DSM e CID. O estudo compara os critérios diagnósticos considerando os domínios: interação social, comunicação e padrão restrito e repetitivo de comportamento, e os respectivos subdomínios. Adicionalmente, são sinalizados instrumentos auxiliares utilizados, tecnologias diagnósticas em desenvolvimento e modelos conceituais que tratam do perfil neuropsicológico.

\section{Diagnóstico de autismo no século XXI}

Os manuais diagnósticos vigentes no século XXI são: DSM-IV-TR e DSM-5, conduzidos pela APA; e CID-10 e CID-11, coordenados pela Organização Mundial de Saúde (OMS) (Figura 1). As últimas alterações, tanto no DSM quanto na CID, foram significativas e têm gerado discussões quanto à sensibilidade e especificidade destes manuais, bem como alterações na prevalência do autismo (Wilson et al., 2013).

Uma alteração importante na segunda revisão da CID-10 foi a diferenciação com o transtorno de linguagem (F80.2), o qual pode ser observado posteriormente no DSM-5 e na CID-11. A linguagem funcional passa a ser central no diagnóstico em detrimento da linguagem estruturada. Ao deslocar o transtorno de linguagem da CID-10 de F84 para F80, a linguagem pragmática da comunicação passa a ser o foco para o diagnóstico.

Os domínios de interação social, comunicação e padrão restrito e repetitivo de comportamento foram mantidos nos diferentes manuais diagnósticos abordados, porém os subdomínios de interação social e comunicação foram reduzidos, enquanto, no padrão restritivo e repetitivo de comportamento, houve uma consolidação no DSM-5 (Peters \& Matson, 2019) do que era vigente nos dois manuais anteriores, CID-10 e DSM-IV-TR (Figura 2). Chama atenção, na CID-11, a ausência do subdomínio "hiper ou hiporreatividade à entrada sensorial ou interesse incomum em aspectos sensoriais do ambiente", embora esta classificação ainda não tenha sido completamente divulgada 
(World Health Organization, 2018). Esse subdomínio estava presente na CID-10 e no DSM-5.

No domínio comunicação, os subdomínios "falta de criatividade e fantasia nos processos de pensamento" e "ausência de brincadeira de faz de conta", presentes na CID-10 e no DSM-IV-TR, estão ausentes no DSM-5 e na CID-11. Uma hipótese é que estes tenham migrado, de forma implícita, para o domínio "padrão restrito e repetitivo de comportamento" por representar inflexibilidade do comportamento.

No DSM-IV-TR (American Psychiatric Association, 2002), vários diagnósticos foram agrupados nos
Transtornos Invasivos de Desenvolvimento (TID). O diagnóstico passa a considerar pelo menos dois dos sintomas na área de interação social, pelo menos um na área de comunicação e pelo menos um na área de comportamentos restritos, repetitivos e estereotipados. Eles separavam, portanto, a interação social da comunicação e enfatizava mais os aspectos sociais do que as estereotipias ou padrões rígidos do comportamento.

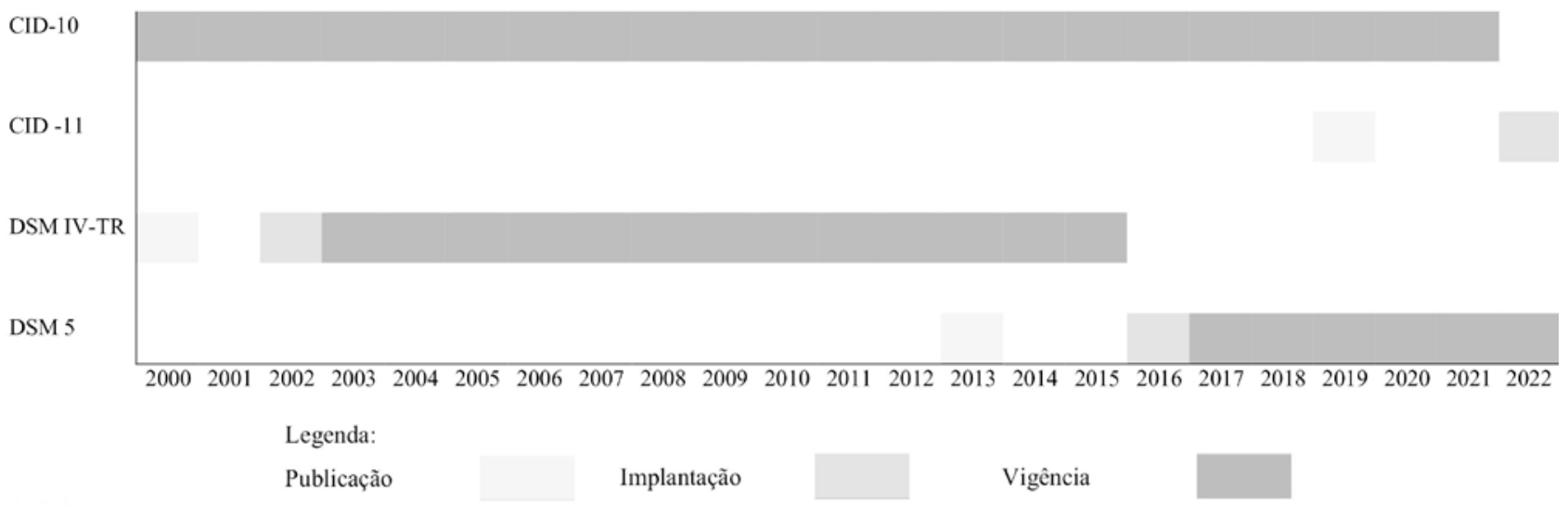

Figura 1. Diagrama temporal dos critérios de diagnóstico do autismo vigentes no século XXI

\begin{tabular}{|c|c|c|c|c|c|c|}
\hline \multicolumn{7}{|c|}{ a) Interação social } \\
\hline \multicolumn{7}{|c|}{ Relações de companheirismo } \\
\hline \multicolumn{7}{|c|}{ Satisfação, interesses ou realizações com outras pessoas } \\
\hline \multicolumn{7}{|c|}{ Reciprocidade social ou emocional } \\
\hline \multicolumn{7}{|c|}{ b) Comunicação } \\
\hline \multicolumn{7}{|c|}{ Falta de criatividade e fantasia nos processos de pensamento } \\
\hline \multicolumn{7}{|c|}{ Deficiências na linguagem oral } \\
\hline \multicolumn{7}{|c|}{ Ausência de brincadeira de faz de conta } \\
\hline \multicolumn{7}{|c|}{ Linguagem estereotipada, repetitiva ou idiossincrática } \\
\hline \multicolumn{7}{|c|}{ Déficits nos processos de desenvolver e manter relacionamentos } \\
\hline \multicolumn{7}{|c|}{ Ausência de comportamentos comunicativos não verbais } \\
\hline \multicolumn{7}{|c|}{ Comunicação social } \\
\hline \multicolumn{7}{|c|}{ c) Padrões restritos e repetitivos de comportamento } \\
\hline \multicolumn{7}{|c|}{$\begin{array}{l}\text { Hiper ou hiporreatividade a entrada sensorial ou interesse incomum em aspectos } \\
\text { sensoriais do ambiente }\end{array}$} \\
\hline \multicolumn{7}{|c|}{ Padrões ritualizados de comportamento verbal ou não-verbal } \\
\hline \multicolumn{7}{|c|}{ Movimentos motores ou uso de objetos } \\
\hline \multicolumn{7}{|c|}{ Aderência excessiva a rotinas } \\
\hline \multicolumn{7}{|c|}{ Resistência excessiva à mudança } \\
\hline \multicolumn{7}{|c|}{ Interesses fixos e restritos } \\
\hline \multirow[t]{4}{*}{ Manuais } & & & CID-10 & $\begin{array}{l}\text { DSM- } \\
\text { IV-TR }\end{array}$ & DSM-5 & CID-11 \\
\hline & \multicolumn{6}{|l|}{ Legenda: } \\
\hline & Presente em um manual & \multicolumn{3}{|c|}{ Presente em três manuais } & & \\
\hline & Presente em dois manuais & \multicolumn{3}{|c|}{ Presente em todos os manuais } & & \\
\hline
\end{tabular}

Figura 2. Diagrama dos domínios e subdomínios do autismo por critérios diagnósticos 
O DSM-5 e a CID-11 entendem o autismo dentro de um único espectro ou categoria, variando em níveis de gravidade, baseado na funcionalidade (DSM-5); ou em níveis de deficiência intelectual e linguagem funcional (CID-11). Além disso, ambos nomeiam o autismo como transtorno do espectro autista (TEA).

O DSM-5 apresenta níveis diferentes relacionados à gravidade do caso, sendo classificados em: a) Nível I - na ausência de apoio, há prejuízo social notável, dificuldades para iniciar interações, por vezes parecem apresentar um interesse reduzido por estas, há tentativas malsucedidas no contato social, além da dificuldade de organização, planejamento e certa inflexibilidade de comportamentos; b) Nível II - exige apoio substancial havendo prejuízos sociais aparentes, limitações para iniciar e manter interações, inflexibilidade de comportamento e dificuldade para lidar com mudanças; c) Nível III - exige muito apoio substancial, havendo déficits graves nas habilidades de comunicação social, inflexibilidade de comportamento e extrema dificuldade com mudanças. Assim, quanto menor o grau de comprometimento do nível, melhor tende a ser o prognóstico do paciente. Já o CID-11 considera, de forma mais clara, a deficiência intelectual e a linguagem funcional, e os diferentes diagnósticos são enquadrados em função do nível de prejuízos nestas habilidades cognitivas.

Em termos gerais, os manuais DSM-IV-TR e CID-10 seguem o seguinte padrão: agrupam as diferentes características do autismo em subgrupos - ou seja, em diferentes diagnósticos com critérios distintos - e usam menos o perfil cognitivo na confirmação diagnóstica. Já os manuais mais recentes (DSM-5 e CID-11) consideram um espectro, adotam não apenas critérios comportamentais e indicadores do desenvolvimento, mas também critérios cognitivos e de adaptação ao meio (funcionalidade/ atividades da vida diária).

Alguns estudos, entretanto, verificam que a mudança dos critérios pode gerar alterações na sensibilidade diagnóstica, especialmente para diagnósticos que não eram enquadrados como autismo, mas que apresentavam prejuízos na comunicação e interação social, como a síndrome de Asperger e os transtornos invasivos do desenvolvimento sem outra especificação (Gibbs, Aldridge, Chandler, Witzlsperger, \& Smith, 2012; Harstad et al., 2015; Wilson et al., 2013). Wilson et al. (2013) verificaram que estes diagnósticos, antes feitos pelo CID-10 ou DSM-IV-TR, apresentaram $44 \%$ de não diagnóstico comparando-se DSM-5 com CID-10, e 22\% não se enquadraram no espectro quando comparados DSM-5 e DSM-IV-TR. Também identifica menor número de diagnóstico em idades mais jovens e diagnósticos mais leves do DSM-IV-TR, como o TID sem outra especificação a partir do DSM-5, quando comparado com DSM-IV-TR (Harstad et al., 2015). Estudo recente (Peters \& Matson, 2019) também corrobora a ideia de que os diagnósticos DSM-IV-TR com critérios menos definidos não são identificados pelo DSM-5. Os autores, porém, ressaltam que, na faixa pré-escolar, a divergência diagnóstica é menor e que, em indivíduos com sintomas mais graves, há mais concordância diagnóstica entre DSM-IV-TR e DSM-5. A sensibilidade da CID-11 ainda não foi observada.

É importante identificar o impacto dessas mudanças no diagnóstico. A retirada do atraso de linguagem, por exemplo, pode aumentar a sensibilidade do diagnóstico dos mais jovens. Destaca-se que, ao se falar em retirada do critério de linguagem, está se falando de linguagem oral, que pode ser critério para outros diagnósticos do neurodesenvolvimento. Entretanto a característica central do TEA está na linguagem pragmática, e não necessariamente na possibilidade da linguagem oral (Mousinho, 2010).

Já a ênfase nos critérios de comportamentos repetitivos e estereotipados, com aumento de um para dois itens desse padrão, pode aumentar a especificidade do diagnóstico. Kent et al (2013) propõem uma flexibilização dos subdomínios encontrados nos dois critérios do DSM-5, construindo um algoritmo diferente para aumentar a sensibilidade do manual. Logo, os critérios de comunicação social não são suficientes para o diagnóstico, quando não há presença de comportamento ritualístico (Peters \& Matson, 2019; Young \& Rodi, 2014).

A mudança do impacto dos critérios de comunicação social pode estar relacionada com as alterações sofridas entre os manuais. Os subdomínios do DSM-IV-TR relacionados à interação social e comunicação foram incorporados a outros subdomínios da reciprocidade socioemocional dos manuais posteriores (DSM-5 e CID-11). Dentre eles são citados a relação de companheirismo, a satisfação e o interesse pelas relações pessoais. Uma das possibilidades para explicar esse englobamento é que, no DSM-IV-TR, alguns subdomínios representavam comportamentos mais descritivos, enquanto as CIDs e o DSM-5 fazem agrupamentos mais conceituais, como o denominado "reciprocidade social emocional" e "comunicação social". Há um movimento similar no DSM-5 em comparação ao CID-11, em que os subdomínios "déficits nos processos de desenvolver e manter relacionamentos", "ausências de comportamentos comunicativos não verbais" e "linguagem estereotipada" são incluídos no domínio comunicação social. No próprio DSM-5 e na CID-11 há uma tendência de agrupamentos mais conceituais e menos descritivos comportamentalmente. Nesses domínios de reciprocidade e comunicação social, são incluídos subdomínios associados a aspectos da linguagem pragmática e desempenho de habilidades sociais, como o ato de conversar adequadamente e gerenciar parcerias sociais.

Além dos critérios englobados, encontram-se subdomínios como ausência de brincadeira de faz de conta, linguagem estereotipada, repetitiva ou idiossincrática e falta de criatividade e fantasia nos processos de pensamento que foram deslocados do domínio de comunicação social para padrões estereotipados no DSM-5. A brincadeira do faz de conta e a criatividade estão mais associadas a padrões de desenvolvimento do pensamento flexível e teoria da mente (habilidade de entender perspectivas diferentes), sendo, portanto, 
mais bem caracterizados nos padrões estereotipados e restritos do pensamento e da ação, enquanto o domínio de comunicação se refere a padrões de desempenho de habilidades sociais e linguagem pragmática.

Nota-se, ainda, que o domínio de padrões repetitivos é o que menos apresenta alterações ao longo do desenvolvimento dos manuais, provavelmente por ser um componente mais consistente no diagnóstico e no próprio constructo cognitivo associado, no que diz respeito aos padrões inflexíveis do pensamento e da ação (Pedersen et al., 2017). Por outro lado, componentes de comunicação social e interação apresentam interface com diferentes constructos cognitivos, como o da linguagem (Tomasello, 2003; Tonietto, Wagner, Trentini, Sperb, \& Parente, 2011), a teoria da mente (Brown, Thibodeau, Pierucci, \& Gilpin, 2017; Smogorzewska, Szumski, \& Grygiel, 2018), padrões de pensamento e socioculturais (Tonietto et al., 2011). O fato de serem permeados por diferentes contingências pode gerar dificuldades na caracterização e definição dos subdomínios, o que justificaria deslocamentos de critérios.

\section{Instrumentos auxiliares}

Os manuais nosológicos despertam questionamentos sobre como mensurar os domínios comportamentais apresentados de forma descritiva. As escalas de avaliação comportamental e a avaliação neuropsicológica nesse cenário possibilitam mensurar objetivamente tais aspectos, avaliando a gravidade e o funcionamento cognitivo. Alguns instrumentos e modelos de avaliação são utilizados junto a esses critérios para auxiliar no diagnóstico (Seize \& Borsa, 2017), como os instrumentos de rastreio, as observações sistematizadas do comportamento (Marques \& Bosa, 2015) e o padrão de desenvolvimento e avaliações do perfil sociocognitivo (Demetriou et al., 2018; Fernandes, Fichman, \& Barros, 2018; Happé \& Frith, 2014).

As escalas de rastreio são utilizadas normalmente para um auxílio diagnóstico precoce, e a proposta é que elas sejam práticas na utilização de apoio diagnóstico. Elas atuam em sintomas que apresentam risco para a confirmação do diagnóstico e podem avaliar populações não clínicas (assintomática) e populações que já apresentam atrasos de desenvolvimento (suspeitas) (Eaves, Wingert, $\&$ Ho, 2006).

Seize e Borsa (2017) realizaram uma revisão sistemática sobre esses instrumentos no Brasil, para rastreio até 36 meses, e encontraram apenas um instrumento traduzido, o Modified Checklist for Autism in Toddlers (M-CHAT), composto por 23 itens e desenvolvido a partir de sintomas presentes no autismo (Losapio \& Pondé, 2006). As autoras o consideram um dos instrumentos mais adequados para rastreio. Algumas outras escalas também validadas no Brasil são: escala de avaliação de traços autísticos (ATA) composta por 23 subescalas divididas em diferentes itens (Assumpção, Kuczynski, Gabriel, \& Rocca, 1999); e a Childhood
Autism Rating Scale (CARS), que compreende quinze itens avaliados em escalonamento de sintomas, o que permite verificar sua gravidade (Pereira, Riesgo, \& Wagner, 2008). Além destes, há a Autism Behavior Chacklist (ABC), desenvolvida a partir de uma escala mais ampla; e a Autism Screening Instrument for Educational Planning (Asiep) (Krug, Arick, \&Almond, 1980). Ela é composta por 57 itens e validada no Brasil por Marteleto e Pedromônico (2005). Contudo sua sensibilidade tem sido criticada em relação à especificidade, pois identifica bem os não autistas, mas não identifica todos os autistas (Fernandes \& Miilher, 2008; Marteleto \& Pedremônico, 2005, Miranda-Linné \& Melin, 2002)

Quanto aos modelos de entrevista/observação desenvolvidos ou validados no Brasil, alguns exemplos são: Protocolo de Avaliação Comportamental para Crianças com Suspeita de Transtorno do Espectro Autista (Protea), um instrumento que utiliza entrevista com pais e a observação de três dimensões do TEA: 1) interação social, linguagem e comunicação, 2) relação com os objetos e brincadeiras; e 3) comportamento estereotipado e autolesivo (Bosa, Zanon, \& Backes, 2016; Marques \& Bosa, 2015); o Autism Diagnostic Observation Schedule (Ados), também composto por entrevista e observação, a partir de quatro módulos correspondentes ao comportamentos de comunicação expressiva e social (Pacífico et al., 2019); e a Autism Diagnostic InterviewRevised (ADIS-R), entrevista com 93 questões referentes aos marcos de desenvolvimento e áreas de sintomatologia do TEA (Becker, 2009). Todos avaliam interação social, atenção compartilhada, brincadeira simbólica, uso de linguagem e exigem maior treinamento de profissionais (Marques \& Bosa, 2015). Nesta mesma linha, destaca-se o protocolo de Indicadores clínicos de Risco para o Desenvolvimento Infantil (Irdi) (Kupfer et al, 2008). Seguindo uma proposta diferente dos outros protocolos, o Irdi identifica risco psíquico no desenvolvimento, dentre eles o autismo. Campana, Lerner \& David (2015), após avaliarem 43 bebês de 18 meses, comparando M-CHAT e Irdi, identificaram que o protocolo Irdi também sinaliza risco para o autismo. O Irdi tem sido muito utilizado em pesquisas, tendo sido incluído na Caderneta de Saúde da Criança (Kupfer \& Bernardino, 2018). É formada por 31 indicadores, observados nas faixas de 0-4 meses, 4-8 meses, 8-12 meses e 12-18 meses (Mota et al., 2015).

Além disso, deve-se considerar a avaliação neuropsicológica, pois a literatura descreve perfis neuropsicológicos condizentes com TEA que envolvem: a) alterações em Teoria da Mente (ToM) no que diz respeito à falsa crença (Baron-Cohen, Leslie, \& Frith, 1985); b) domínios de funções executivas, como memória de trabalho (Barendse et al., 2018; Faja \& Dawson, 2014), controle inibitório (Schmitt, White, Cook, Sweeney, \& Mosconi, 2018) e flexibilidade cognitiva (Leung \& Zakzanis, 2014).

Neste contexto, as funções executivas junto ao funcionamento cognitivo global (QI) caracterizariam 
o perfil cognitivo e auxiliariam na identificação de gravidade dos sintomas (Constantino \& Charman, 2016; Polyak, Kubina, \& Girirajan, 2015), mas não conseguiriam definir de forma sensível o diagnóstico (Fernandes et al., 2018; Losh et al., 2009).

\section{Tecnologias diagnósticas em desenvolvimento}

O diagnóstico precoce do autismo possibilita intervenções mais cedo, favorecendo o desenvolvimento das habilidades comprometidas e proporcionando uma adaptação melhor para o indivíduo e sua família. $\mathrm{O}$ diagnóstico em idades cada vez mais precoces, entretanto, é um desafio. A Academia Americana de Pediatria (AAP) preconiza que seja realizada vigilância, estando alerta às queixas familiares e executando rastreamento por meio da utilização de instrumentos padronizados para avaliação do risco (James, Pizur-Barnekow, \& Schefkind, 2014). Os critérios de autismo no DSM-IV-R foram validados para crianças a partir de três anos de idade, todavia há literatura indicando a possibilidade de diagnóstico em crianças mais novas, com ênfase na importância das intervenções precoces. Neste contexto, marcadores biológicos que antecipem as manifestações comportamentais têm sido objetos de estudos. Yang et al. (2018) identificaram proteínas que podem ser biomarcadores no sangue; Chen, Yang, Wu, Chuang, \& Huang (2019) avaliaram a relação de TEA com biomarcadores de imagens de ressonância magnética, enquanto outro estudo (Del Valle Rubido et al., 2018) avaliou diversos biomarcadores e sua correlação com medidas de avaliação clínica. Contudo ainda não existem marcadores biológicos comprovados para o diagnóstico do autismo.

Técnicas computacionais também vêm sendo utilizadas para identificar anormalidades em áreas do cérebro, utilizando modelos preditivos em imagens de ressonância magnética (Li, Liu, Sun, Shen, \& Wang, 2018) ou eletroencefalograma (Bosl, Tager-Flusberg, \& Nelson, 2018). Outra possibilidade são as técnicas que combinam informações de diferentes fontes de avaliação para melhorar o desempenho preditivo (Zhao, Qiao, Shi, Yap, \& Shen, 2017). As abordagens incluem a tecnologia de rastreamento ocular para avaliar a preferência por imagens geométricas dinâmicas em crianças com idade entre 14 e 42 meses, sugerindo um tempo de fixação maior nestas imagens em crianças com TEA (Pierce, Conant, Hazin, Stoner, \& Desmond, 2011).

\section{Considerações finais}

Este artigo aborda a evolução do diagnóstico do autismo com base em manuais diagnósticos. Verificam-se as alterações no percurso desse diagnóstico primeiro no contexto psiquiátrico até sua definição como transtorno do neurodesenvolvimento infantil. Entender as mudanças e a evolução desses critérios dá margem a discussões quanto às possibilidades de impacto na epidemiologia do transtorno, bem como diferentes instrumentos que podem compor e apurar o processo de avaliação, como utilização de escalas, avaliações cognitivas e novas tecnologias.

Vários estudos discutem diferenças na identificação do diagnóstico, em especial quando se comparam DSM-IV-TR e DSM-5. Estes estudos não são totalmente convergentes em seus dados, mas mostram, de forma um pouco mais consistente, que o DSM-5 identifica menor número de diagnósticos quando comparado com o DSM-IV-TR, talvez em função da maior sensibilidade de seus critérios. O DSM-5 não identifica da mesma forma os outros diagnósticos que não cumpriam todos os critérios para autismo existentes no DSM-IV-TR, como o TID sem outra especificação ou diagnósticos com sintomas mais leves. Entretanto esses índices de identificação se aproximam na faixa pré-escolar, talvez em função dos sintomas nessa faixa etária serem mais evidentes. Além disso, houve mudanças importantes no critério de comunicação social, com englobamentos ou deslocamentos de sintomas e maior ênfase no critério de movimentos ritualísticos/estereotipados.

Em relação ao processo de avaliação, os novos critérios podem se beneficiar de outros instrumentos, como escalas, que esclarecem melhor determinados comportamentos, e as novas tecnologias, que podem trazer melhor objetividade ao diagnóstico clínico. Mas também serão necessárias, considerando os novos critérios cognitivos citados pelo CID-11, avaliações cognitivas de linguagem e de funcionamento cognitivo global. $\mathrm{O}$ DSM-5 já traz os déficits cognitivos como comorbidades que implicam na gravidade dos sintomas, contudo o CID11 aponta o funcionamento cognitivo como critérios de subclassificações dentro do espectro.

Compreender a evolução dos critérios diagnósticos tende a promover desenvolvimento da avaliação clínica no que diz respeito à construção de algoritmos cada vez mais apurados e consistentes. Isso potencializa a antecipação do diagnóstico e as intervenções necessárias para um melhor prognóstico.

\section{Autism diagnosis in the 21 st century: evolution of subdomains in nosological categorizations}

Abstract: The criteria that subsidized the diagnosis of autism have undergone several changes over the years, following the hegemonic conceptual assumptions of the period. This study analyzed the evolution of the diagnosis of autism during the 21st century with use of the domains and subdomains that served as basis for nosological categorizations. Additionally, the auxiliary instruments used, some diagnostic technologies under development, and conceptual models that address neuropsychological profiles are presented. This documentary research utilized diagnostic manuals of the period. The domains of social interaction, 
communication, and restricted and repetitive behavior patterns were maintained in the different diagnostic manuals addressed. The subdomains of social interaction and communication were reduced, while restricted and repetitive pattern was consolidated in DSM-5. Understanding the evolution of diagnostic criteria tends to promote clinical development, potentiating the anticipation of the diagnosis and the interventions necessary for a better prognosis.

Keywords: autism spectrum disorder, autistic disorder, diagnosis, Diagnostic and Statistical Manual of Mental Disorders, International Classification of Diseases.

\section{Diagnostic de l'autisme au 21 e siècle : évolution des domaines dans les catégorisations nosologiques}

Résumé : Les critères qui subsidient le diagnostic de l'autisme ont subi plusieurs changements, suivant les hypothèses conceptuelles hégémoniques de la période. Cette étude vise à analyser l'évolution du diagnostic de l'autisme au 21 e siècle, à partir des domaines et sous-domaines sur lesquelles reposent les catégorisations nosologiques. En outre, les instruments auxiliaires utilisés et certaines technologies de diagnostic en cours de développement sont signalés, en plus des modèles conceptuels sur le profil neuropsychologique. Il s'agit d'une recherche documentaire, basée sur les manuels de diagnostic existant à l'époque. Les domaines de l'interaction sociale, de la communication et des comportements restreints et répétitifs ont été maintenus dans les manuels de diagnostic abordés. Les sous-domaines de l'interaction sociale et de la communication ont été réduits, tandis que le norme restreint et répétitif a été consolidé dans le DSM-5. Comprendre l'évolution des critères diagnostiques tend à favoriser le développement clinique, l'anticipation du diagnostic, et les interventions nécessaires à un meilleur pronostic.

Mots-clés : trouble du spectre autistique, trouble autistique, diagnostic, Manuel Diagnostique et Statistique des Troubles Mentaux, Classification Internationale des Maladies.

\section{Diagnóstico del autismo en el siglo XXI: evolución de dominios en categorizaciones nosológicas}

Resumen: Los criterios que ayudaron el diagnóstico de autismo han sufrido varios cambios, siguiendo los supuestos conceptuales hegemónicos en el período. El objetivo de este estudio es analizar la evolución del diagnóstico de autismo en el siglo XXI, en función de los dominios y subdominios que se basan las categorizaciones nosológicas. Además, se señalan los instrumentos auxiliares utilizados, algunas tecnologías de diagnóstico en desarrollo y modelos conceptuales que abordan el perfil neuropsicológico. Esta es una investigación documental, basada en los manuales de diagnóstico existentes en el período. Los dominios de interacción social, comunicación y patrones de conducta restringidos y repetitivos se mantuvieron en los manuales de diagnóstico abordados. Los subdominios de interacción social y comunicación se redujeron, mientras que el patrón restringido y repetitivo se consolidó en el DSM-5. Comprender la evolución de los criterios de diagnóstico tiende a promover el desarrollo clínico, fomentando la anticipación diagnóstica e intervenciones necesarias para un mejor pronóstico.

Palabras clave: trastorno del espectro autista, trastorno autista, Diagnóstico, Manual Diagnóstico y Estadístico de los Trastornos Mentales, Clasificación Internacional de Enfermedades.

\section{Referências}

Aguiar, M. P., \& Ortega, F. J. G. (2017). Psiquiatria biológica e psicofarmacologia: a formação de uma rede tecnocientífica. Physis: Revista de Saúde Coletiva, 27(4), 889-910. doi: 10.1590/s0103-73312017000400003

American Psychiatric Association. (1952). Diagnostic and Statistical Manual: Mental Disorders. DSM-I. Washington, DC: APA.

American Psychiatric Association. (1968). Diagnostic and Statistical Manual of Mental Disorders. DSM-II. Washington, DC: APA. Recuperado de https://bit.ly/2R61tWp
American Psychiatric Association. (2002). DSM IV - TR Manual de Diagnóstico e Estatístico das Perturbações Mentais (4a ed.). Porto Alegre, RS: Artmed.

American Psychiatric Association. (2014). Manual Diagnóstico e Estatístico de Transtornos Mentais: DSM-5 (5a ed.). Porto Alegre, RS: Artmed.

Araújo, A. C., \& Neto, F. L. (2014). A nova Classificação Americana Para os Transtornos Mentais - o DSM-5. Revista Brasileira de Terapia Comportamental $e$ Cognitiva, 16(1), 62-87.

Assumpção, F. B., Jr, Kuczynski, E., Gabriel, M. R., \& Rocca, C. C. (1999). Escala de avaliação de traços autísticos (ATA): 
validade e confiabilidade de uma escala para a detecção de condutas artísticas. Arquivos de Neuro-Psiquiatria, 57(1), 23-29. doi: 10.1590/S0004-282X1999000100005

Assumpção, F. B., Jr., \& Pimentel, A. C. M. (2000). Autismo infantil. Revista Brasileira de Psiquiatria, 22(Supl I), 37-39.

Barendse, E. M., Schreuder, L. J., Thoonen, G., Hendriks, M. P. H., Kessels, R. P. C., Backes, W. H. . . Jansen, J. F. A. (2018). Working memory network alterations in high-functioning adolescents with an autism spectrum disorder: FMRI correlates for memory in ASD. Psychiatry and Clinical Neurosciences, 72(2), 73-83. doi: 10.1111/pcn.12602

Baron-Cohen, S., Leslie, A. M., \& Frith, U. (1985). Does the autistic child have a "theory of mind" ? Cognition, 21(1), 37-46. doi: 10.1016/0010-0277(85)90022-8

Becker, M. M. (2009). Tradução e validação da entrevista Autism Diagnostic Interview-Revised (Adis-R) (Dissertação de mestrado). Universidade Federal do Rio Grande do Sul, Porto Alegre, RS.

Benedicto, R. P., Wai, M. F. P., Oliveira, R. M., Godoy, C., \& Costa, M. L., Jr. (2013). Análise da evolução dos transtornos mentais e comportamentais ao longo das revisões da classificação internacional de doenças. SMAD: Revista Eletrônica Saúde Mental, Ácool e Drogas, 9(1), 28-32.

Bosa, C, A,; Zanom, R., B., \& Backes, B. (2016). Autismo: construção de um Protocolo de Avaliação do Comportamento da Criança - Protea R. Revista Psicologia: Teoria e Prática, 18(1), 194-205. doi: 10.15348/1980-6906/psicologia.v18n1p194-205

Bosl, W. J., Tager-Flusberg, H., \& Nelson, C. A. (2018). EEG analytics for early detection of autism spectrum disorder: a data-driven approach. Scientific Reports, 8(1), 6828. doi: 10.1038/s41598-018-24318-x

Brown, M. M., Thibodeau, R. B., Pierucci, J. M., \& Gilpin, A. T. (2017). Supporting the development of empathy: the role of theory of mind and fantasy orientation. Social Development, 26(4), 951-964. doi: 10.1111/sode.12232

Campana, N. T. C., Lerner, R., \& David, V. F. (2015). CDRI as an instrument to evaluate infants with developmental problems associated with autism. Paidéia (Ribeirão Preto), 25(60), 85-93. doi: 10.1590/1982-43272560201511

Chen, C.-M., Yang, P., Wu, M.-T., Chuang, T.-C., \& Huang, T.-Y. (2019). Deriving and validating biomarkers associated with autism spectrum disorders from a largescale resting-state database. Scientific Reports, 9(1), 9043. doi: 10.1038/s41598-019-45465-9

Constantino, J. N., \& Charman, T. (2016). Diagnosis of autism spectrum disorder: Reconciling the syndrome, its diverse origins, and variation in expression. The Lancet Neurology, 15(3), 279-291. doi: 10.1016/S1474-4422(15)00151-9

Del Valle Rubido, M., McCracken, J. T., Hollander, E., Shic, F., Noeldeke, J., Boak, L. . . . Umbricht, D. (2018). In search of biomarkers for autism spectrum disorder: exploratory biomarkers in autism. Autism Research, 11(11), 1567-1579. https://doi.org/10.1002/aur.2026
Demetriou, E. A., Lampit, A., Quintana, D. S., Naismith, S. L., Song, Y. J. C., Pye, J. E. . . Guastella, A. J. (2018). Autism spectrum disorders: a meta-analysis of executive function. Molecular Psychiatry, 23(5), 1198-1204.

Dunker, C. I. L. (2014). Questões entre a psicanálise e o DSM. Jornal de Psicanálise, 47(87), 79-107.

Eaves, L. C., Wingert, H., \& Ho, H. H. (2006). Screening for autism: agreement with diagnosis. Autism, 10(3), 229-242. doi: 10.1177/1362361306063288

Facion, J. R. (2005). Transtornos invasivos do desenvolvimento e transtornos de comportamento disruptivo (2a ed.). Curitiba, PR: IBPEX.

Faja, S., \& Dawson, G. (2014). Performance on the dimensional change card sort and backward digit span by young children with autism without intellectual disability. Child Neuropsychology, 20(6), 692-699. doi: 10.1080/09297049.2013.856395

Fernandes, C. S., Fichman, H. C., \& Barros, P. S. (2018). Evidências de diagnóstico diferencial entre transtorno do espectro autista (TEA) e transtorno do desenvolvimento intelectual (TDI): análise de casos. Revista Neuropsicologia Latinoamericana, 10(2), 29-41.

Fernandes, F. D. M., \& Miilher, L. P. (2008). Relações entre a Autistic Behavior Checklist (ABC) e o perfil funcional da comunicação no espectro autístico. Pró-Fono: Revista de Atualização Científica, 20(2), 111-116. doi: 10.1590/S0104-56872008000200007

Gadia, C. A., Tuchman, R., \& Rotta, N. T. (2004). Autismo e doenças invasivas de desenvolvimento. Jornal de Pediatria, 80, S83-94.

Gibbs, V., Aldridge, F., Chandler, F., Witzlsperger, E., \& Smith, K. (2012). Brief report: an exploratory study comparing diagnostic outcomes for autism spectrum disorders under DSM-IV-TR with the Proposed DSM-5 revision. Journal of Autism and Developmental Disorders, 42(8), 1750-1756. doi: 10.1007/s10803-012-1560-6

Happé, F., \& Frith, U. (2014). Annual research review: towards a developmental neuroscience of atypical social cognition. Journal of Child Psychology and Psychiatry, 55(6), 553-577. doi: 10.1111/jcpp. 12162

Harstad, E. B., Fogler, J., Sideridis, G., Weas, S., Mauras, C., \& Barbaresi, W. J. (2015). Comparing diagnostic outcomes of autism spectrum disorder using DSMIV-TR and DSM-5 criteria. Journal of Autism and Developmental Disorders, 45(5), 1437-1450. doi: 10.1007/s10803-014-2306-4

Huppke P, Laccome F, Krämer N, Engel W, \& Hanefeld F. (2000). Rett syndrome: analysis of MeCP2 and clinical characterization of 31 patients. Human Molecular Genetics, 9(9),1369-1375. doi: 10.1093/hmg/9.9.1369

James, L. W., Pizur-Barnekow, K. A., \& Schefkind, S. (2014). Online survey examining practitioners' perceived preparedness in the early identification of autism. American Journal of Occupational Therapy, 68(1), e13-e20. doi: 10.5014/ajot.2014.009027

Kanner, L. (1943). Autistic disturbances of affective contact. Nervous Child, 2, 217-250. 
Kent, R. G., Carrington, S. J., Le Couteur, A., Gould, J., Wing, L., Maljaars, J. . . . Leekam, S. R. (2013). Diagnosing autism spectrum disorder: who will get a DSM-5 diagnosis? Journal of Child Psychology and Psychiatry, 54(11), 1242-1250. doi: 10.1111/jcpp.12085

Krug, D., Arick, J., \& Almond, P. (1980). Behavior checklist for identifying severely handicapped individuals with high levels of autistic behavior. Journal of Child Psychology and Psychiatry, 21, 221-229. doi: 10.1111/j.1469-7610.1980.tb01797.x

Kupfer, M. C. M., Bernardino, L. M. F., Jerusalinsky, A. F., Rocha, P. S. B., Lerner, R., \& Pesaro, M. E. (2008). A pesquisa IRDI: resultados finais. In R. Lerner \& M. C. M. Kupfer (Orgs.), Psicanálise com crianças: clínica e pesquisa (pp. 221-230). São Paulo, SP: Escuta.

Kupfer, M. C. M., \& Bernardino, L. M. F. (2018). IRDI: um instrumento que leva a psicanálise à polis. Estilos da Clínica, 23(1), 62-82. doi: 10.11606/issn.1981-1624.v23i1p62-82

Leung, R. C., \& Zakzanis, K. K. (2014). Brief report: cognitive flexibility in autism spectrum disorders: a quantitative review. Journal of Autism and Developmental Disorders, 44(10), 2628-2645. doi: 10.1007/s10803-014-2136-4

Li, G., Liu, M., Sun, Q., Shen, D., \& Wang, L. (2018). Early diagnosis of autism disease by multi-channel CNNs. In Y. Shi, H.-I. Suk, \& M. Liu (Eds.), Machine learning in medical imaging (Vol. 11.046, pp. 303-309). New York: Springer. doi: 10.1007/978-3-030-00919-9_35

Losapio, M F, \& Pondé, M P. (2008). Tradução para o português da escala M-CHAT para rastreamento precoce de autismo. Revista de Psiquiatria do Rio Grande do Sul, 30(3), 221-229. doi: 10.1590/S0101-81082008000400011

Losh, M., Adolphs, R., Poe, M. D., Couture, S., Penn, D., Baranek, G. T. . . Piven, J. (2009). Neuropsychological profile of autism and the broad autism phenotype. Archives of General Psychiatry, 66(5), 518. doi: 10.1001/archgenpsychiatry.2009.34

Marques, D. F., \& Bosa, C. A. (2015). Protocolo de avaliação de crianças com autismo: evidências de validade de critério. Psicologia: Teoria e Pesquisa, 31(1), 43-51. doi: 10.1590/0102-37722015011085043051

Marteleto, M. R. F., \& Pedromônico, M R M. (2005). Validity of Autism Behavior Checklist (ABC): preliminary study. Brazilian Journal of Psychiatry, 27(4), 295-301. doi: 10.1590/S1516-44462005000400008

Martinhago, F., \& Caponi, S. (2019). Controvérsias sobre o uso do DSM para diagnósticos de transtornos mentais. Physis: Revista de Saúde Coletiva, 29(2), 1-19.

Miranda-Linné, F. M., \& Melin, L. (2002) A factor analytic study of the Autism Behavior Checklist. Journal of Autism Developmental Disease, 32(3), 181-188.

Mota, A. Di-P., Lerner, R., Escobar, A. M. U., Kupfer, M. C. M., Rocha, F. M. M., \& Santos, L S. (2015). Associação entre sinais de sofrimento psíquico até dezoito meses e rebaixamento da qualidade de vida aos seis anos de idade. Psicologia USP, 26(3), 464-473. doi: 10.1590/0103-656420130043
Mousinho, R. (2010). O falanteinocente: linguagem pragmática e habilidades sociais no autismo de alto desempenho. Revista de Psicopedagogia, 27(84), 385-394.

Neumann, D. M. C., Tariga, A. R., Perez, D. F., Gomes, P. M., Silveira, J. S., \& Azambuja, L. S. (2017). Avaliação neuropsicológica do transtorno do espectro autista. Psicologia.Pt. Recuperado de https://bit.ly/325uKqI

Pacífico, M. C., de Paula, C. S., Namur, V.S., Lowenthal, R., Bosa, C. A., \& Teixeira, M. C. T. V. (2019). Preliminary evidence of the validity process of the Autism Diagnostic Observation Schedule (ADOS): translation, cross-cultural adaptation and semantic equivalence of the Brazilian Portuguese version. Trends in Psychiatry and Psychotherapy, 41(3), 218-226. doi: 10.1590/2237-6089-2018-0063

Pedersen, A. L., Pettygrove, S., Lu, Z., Andrews, J., Meaney, F. J., Kurzius-Spencer, M. . . Cunniff, C. (2017). DSM criteria that best differentiate intellectual disability from autism spectrum disorder. Child Psychiatry \& Human Development, 48(4), 537-545. doi: 10.1007/s10578-016-0681-0

Pereira, A., Riesgo, R. S., \& Wagner, M. B. (2008). Autismo infantil: Tradução e validação da Childhood Autism Rating Scale para uso no Brasil. Jornal de Pediatria, 84(6), 487-494. doi: 10.1590/S0021-75572008000700004

Peters, W. J., \& Matson, J. L. (2019). Comparing rates of diagnosis using DSM-IV-TR versus DSM-5 criteria for autism spectrum disorder. Journal of Autism and Developmental Disorders, 50, 1898-1906. doi: 10.1007/s10803-019-03941-1

Pierce, K., Conant, D., Hazin, R., Stoner, R., \& Desmond, J. (2011). Preference for geometric patterns early in life as a risk factor for autism. Archives of General Psychiatry, 68(1), 101. doi: 10.1001/archgenpsychiatry.2010.113

Polyak, A., Kubina, R. M., \& Girirajan, S. (2015). Comorbidity of intellectual disability confounds ascertainment of autism: implications for genetic diagnosis. American Journal of Medical Genetics Part B: Neuropsychiatric Genetics, 168(7), 600-608. doi: 10.1002/ajmg.b.32338

Schmitt, L. M., White, S. P., Cook, E. H., Sweeney, J. A., \& Mosconi, M. W. (2018). Cognitive mechanisms of inhibitory control deficits in autism spectrum disorder. Journal of Child Psychology and Psychiatry, 59(5), 586-595. doi: 10.1111/jcpp. 12837

Seize, M. M., \& Borsa, J. C. (2017). Instrumentos para rastreamento de sinais precoces do autismo: revisão sistemática. Psico-USF, 22(1), 161-176. doi: 10.1590/1413-82712017220114

Smogorzewska, J., Szumski, G., \& Grygiel, P. (2018). Same or different? Theory of mind among children with and without disabilities. PLOS ONE, 13(10), e0202553. doi: 10.1371/journal.pone. 0202553

Stelzer, F. G. (2010). Uma pequena história do autismo (Vol. 1). São Leopoldo, RS: Associação Mantenedora Pandorga.

Tomasello, M. (2003). Constructing a language: a usagebased theory of language acquisition. Cambridge, MA: Harvard University Press. 
Tonietto, L., Wagner, G. P., Trentini, C. M., Sperb, T. M., \& Parente, M. A. M. P. (2011). Interfaces entre funções executivas, linguagem e intencionalidade. Paidéia (Ribeirão Preto), 21(49), 247-255. doi: 10.1590/S0103-863X2011000200012

Vargas, R. M., \& Schmidt, C. (2011). Autismo e esquizofrenia: compreendendo diferentes condições. Recuperado de https://bit.ly/3307DwM

Whitaker, R. (2017). Anatomia de uma epidemia: pílulas mágicas, drogas psiquiátricas e o aumento assombroso da doença mental. Manguinhos, RJ: Fiocruz.

Wilson, C. E., Gillan, N., Spain, D., Robertson, D., Roberts, G., Murphy, C. M. . . . Murphy, D. G. M. (2013). Comparison of ICD-10R, DSM-IV-TR and DSM-5 in an adult autism spectrum disorder diagnostic clinic. Journal of Autism and Developmental Disorders, 43(11), 2515-2525. doi: 10.1007/s10803-013-1799-6

World Health Organization. (2018). ICD-11 for Mortality and Morbidity Statistics. Geneva: WHO. Recuperado de https://bit.ly/35gOVnE
Yang, J., Chen, Y., Xiong, X., Zhou, X., Han, L., Ni, L. . . Huang, C. (2018). Peptidome analysis reveals novel serum biomarkers for children with autism spectrum disorder in China. Proteomics: Clinical Applications, 12(5), 1700164. doi: 10.1002/prca.201700164

Young, R. L., \& Rodi, M. L. (2014). Redefining autism spectrum disorder using DSM-5: the implications of the proposed DSM-5 criteria for autism spectrum disorders. Journal of Autism and Developmental Disorders, 44(4), 758-765. doi: 10.1007/s10803-013-1927-3

Zhao, F., Qiao, L., Shi, F., Yap, P.-T., \& Shen, D. (2017). Feature fusion via hierarchical supervised local CCA for diagnosis of autism spectrum disorder. Brain Imaging and Behavior, 11(4), 1050-1060. doi: 10.1007/s11682-016-9587-5

Recebido: $14 / 02 / 2020$

Revisado: 20/07/2020

Aprovado: 22/09/2020 